\title{
Thermographic method for quantifying in-plane non-uniform paper drying
}

\begin{abstract}
* RISE Bioeconomy, Drottning Kristinas väg 61 114 86, Stockholm, Sweden, aron.tysen@ri.se
\end{abstract}
** Karlstad University, Karlstad, Sweden

\begin{abstract}
Arial, 9pt, bold)
A thermographic method has been developed, in order to study in-plane non-uniformity during through air drying of paper tissue products. Non-uniformity in drying of paper is unavoidable, due to the heterogeneous nature of the product. It can be detrimental to the quality of the product, and significantly lower production capacity. The method utilises the endothermic nature of the drying process to quantify non-uniformities. The temperature varies with amount of remaining water. The method is here applied to through air drying of tissue paper, but could also be used for conventional contact drying of other paper grades.
\end{abstract}

\section{Introduction (Arial, 9pt, bold)}

The production of paper is the continuous process of dispersing a solution of water, disintegrated wood fibres and optional additives on a forming fabric, where the water is then removed in several steps. First there is gravitational dewatering, then mechanical dewatering by pressing, and finally thermal drying. After this the fibre web is wound up on huge rolls and sent to converters, where it is cut and prepared into the final product. The fundamentals of this process have remained the same through history, with modifications to increase production speed and capacity, to increase the output. A modern paper machine may be several meters wide and run at $>2000 \mathrm{~m} / \mathrm{min}$ [1].

Most modern paper products adhere to these process steps, but there are notable exceptions. Tissue paper, such as toilet paper, towel paper, napkins and such are in many ways unique products. They are considerably lighter than most other products and have very specific properties to suit their intended usage. Important properties may be water absorption capacity, softness or wet strength. Premium grade tissue products are generally produced on special paper machines, where the pressing step of papermaking is omitted, and the thermal drying is carried out by through air drying (TAD). The mechanical pressing is a very energy efficient way of removing water, but cannot reach higher than 40-45 \% dryness, i.e. the percentage of dry fibre weight in relation to the total weight of the remaining water and the dry fibres combined. It also heavily densifies the paper, which is detrimental to the softness and water absorption properties. The thermal drying, usually achieved by contact drying on steam heated cylinders, is much more energy intensive and responsible for removing the last remaining water. This water is located in the fibre walls and to varying degrees chemically bound to the fibres, requiring significantly more energy to be evaporated $[2,3,4]$.

The TAD process, with the lack of wet pressing, initiates the thermal drying at much lower dryness ( 24 \%) and exchange the contact drying with convection drying with hot air permeating the wet paper web. This allows for very high drying rates as the drying medium (the hot air) is in direct contact with the moist fibres. However, the air is heated by burning natural gas, and the specific energy required is up to $30 \%$ higher than for conventional paper drying. All these considerations combined means that the cost of the water removal is significantly higher than for other processes [5, 4].

The fibre web is also a heterogeneous material with varying degrees of non-uniformity in mass distribution of fibres (i.e. local variations of grammage; weight per square meter) and moisture distribution. The local air flow through the wet web is influenced by these variations, and would thus presumably lead to non-uniform drying as the supply of energy for drying is directly connected to the local air flow. The degree of variation in spatial fibre mass distribution is called formation, where high formation numbers indicate larger local variations (resulting in unwanted local variations in product properties) [6].

A more indirect reason for drying non-uniformity is the interaction between the fibre web and the underlying fabric. The fabric is a weave of polymeric yarns in different configurations to obtain a structure suitable for whichever product should be produced. The degree of contact between the fibre web and the fabric is a fundamental parameter for the development of the intended paper properties [7].

The emissivity of paper during drying is a complex issue, due to the porous nature of the material, the transient decreasing dryness, and the surface properties. Hyll [8] performed an extensive investigation on the emissivity of paper. The dryness was found to influence the emissivity, especially as the dryness got below $50 \%$ and downwards. It typically ranges between 0.7 and 0.9 for the dryness changes relevant for the paper drying process.

\section{Materials and method}

An experimental set-up was developed where paper samples were placed on a permeable surface connected to a fan. The fan (an industrial vacuum cleaner) enabled air to be drawn through the sample and fabric, thus drying the sample by through air drying. An infrared camera mounted perpendicular to the sample captured the spatial surface temperature 
of the sample during the drying sequence. Sketches of the drying equipment and the sample placement are seen in figure 1 and figure 2.

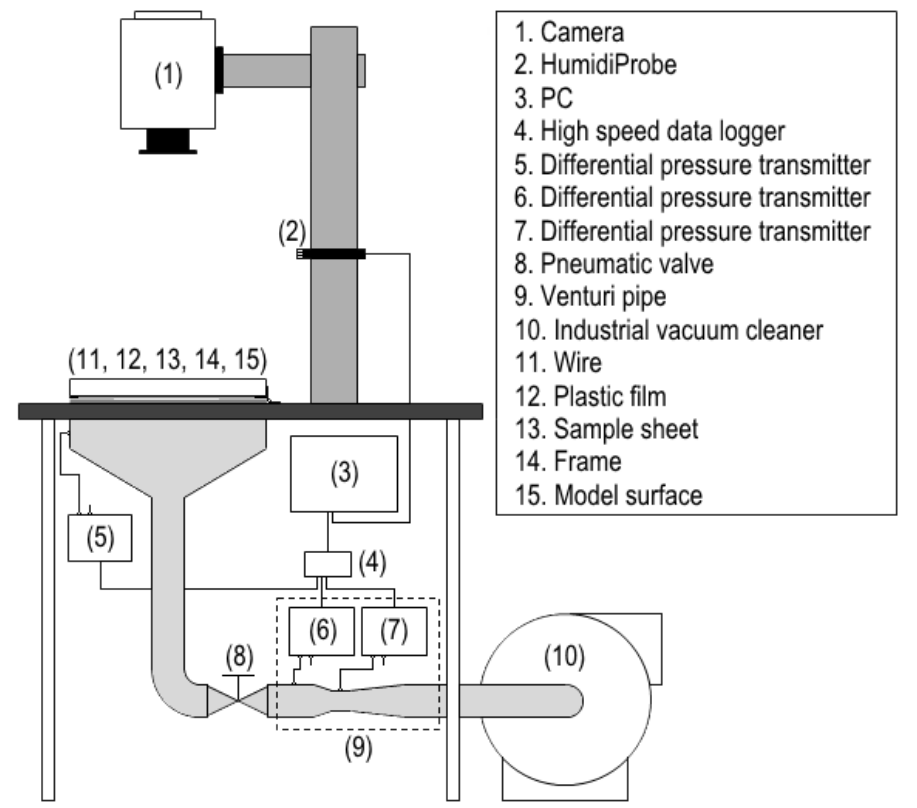

Fig 1. Experimental set-up, with infrared camera perpendicular to sample (dimensions are not to scale).

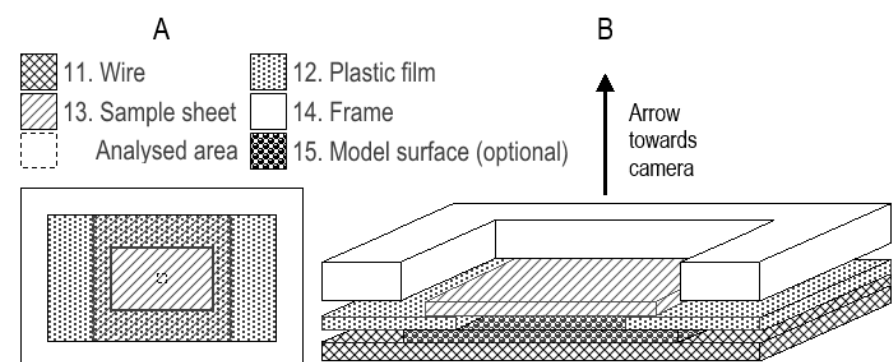

Fig 2. Top-view $(A)$ and cross section $(B)$ of the sample placement, also indicating the analysed area (dimensions are not to scale).

Wet paper samples (13) were placed on the permeable surface (11), which was either a paper forming wire, one of two commercial TAD moulding fabrics, or a model surface (metallic sheet with well-defined holes with a diameter of 2 $\mathrm{mm}$ ). An impermeable plastic film (12) with an opening was placed between the sample and the permeable surface to control the area exposed to air flow. The opening was either 25 or $100 \mathrm{~cm}^{2}$, depending on some sample properties. A frame (14) restrained the sample in one dimension. A pneumatic valve (8) was used to control when drying was initiated. Pressure transmitters $(6,7)$ connected to a Venturi pipe (9) indirectly measured the air flow, while the pressure drop over sample and permeable surface was also measured (5). The relative humidity and the air temperature were measured with a HumidiProbe (2). An infrared camera (1) was mounted perpendicular to the sample arrangement. The measuring equipment was connected to a computer (3) where all logging was done. The analog signal of the pressure transmitters was first converted to digital through a data acquisition system (4).

The spatial transient surface temperature readings were used to evaluate influence of paper formation and the fibre web-fabric interaction on the non-uniformity of through air drying.

The method is described in detail in Tysén [9], Tysén and Vomhoff [10].

\subsection{Thermographic method}

The infrared camera used for this trial was a FLIR ThermoVision ${ }^{\text {TM }}$ SC6000 HS. It has a $640 \times 512$ cooled photometric detector. It detects mid-wave infrared radiation in the 3-5 $\mu \mathrm{m}$ range. The software FLIR ResearchIR Max 4.40.6.24 was used to record the drying sequence. Parameters set in the software are shown in table 1.

Table 1. Parameters set in the thermography software.

\begin{tabular}{|l|l|}
\hline Emissivity & 0.8 \\
\hline Distance [m] & 0.3 \\
\hline
\end{tabular}




\begin{tabular}{|l|l|}
\hline Reflected temperature $\left[{ }^{\circ} \mathrm{C}\right]$ & 24.5 \\
\hline Atmospheric temperature $\left[{ }^{\circ} \mathrm{C}\right]$ & 22.8 \\
\hline Relative humidity $[\%]$ & 54 \\
\hline Frame rate $[\mathrm{Hz}]$ & 10 \\
\hline
\end{tabular}

The emissivity was chosen in accordance with values reported in the literature. Since the emissivity changes with the dryness of the paper sample but was set as a constant in the software, there was inherently a slight error in the reported temperature. The value was chosen to be representative of the emissivity in the dryness range where an erroneous value would have the biggest impact. In the evaluation of the temperature data, only the relative transient temperature change was used, which significantly reduced the impact of the deviation in emissivity on the results of the evaluation.

The reflected temperature was measured by placing a high-reflectance gold surface with Lambertian scattering distribution where the sample would be and setting emissivity to unity.

Atmospheric temperature and relative humidity was obtained from the HumidiProbe.

For the investigation of formation, a $25 \mathrm{~mm}$ lens was used. For the investigation of fibre web-fabric interaction a $100 \mathrm{~mm}$ lens fitted with distances was used. The respective resulting resolutions were $0.28 \mathrm{~mm}^{2}$ and $0.079 \mathrm{~mm}^{2}$ per pixel.

The method utilises the endothermic nature of evaporation of water. The energy needed to achieve the evaporation will decrease the temperature. As the drying progresses the temperature is slowly increased since there spatially is less ongoing drying as areas preferentially dries out. When the drying is finished the temperature levels out close to the temperature of the drying air. The non-uniformity of spatial moisture distribution and air flow both contribute to pixels reaching the temperature plateau at different moments in time. With this in mind, the time it took for a specific pixel to reach the temperature plateau was used to quantify local drying times. Six semi-arbitrarily chosen frames from a representative sequence are seen in figure 3.


Fig 3. Six frames from a representative sequence.

The first frame (A) was immediately prior to initiating drying. The second frame $(B)$ is when the minimum temperature was reached, when the entire analysed area is still drying. The third to fifth frame (C-E) are during the increasing temperature period, where the non-uniformity became prevalent. The sixth frame $(F)$ is when drying was finished and a uniform temperature level was reached.

When analysing the transient pixel temperature, a median filter was applied to decrease the influence of high frequency noise in the signal. A representative example of transient pixel temperature with and without filtering is shown in figure 4.

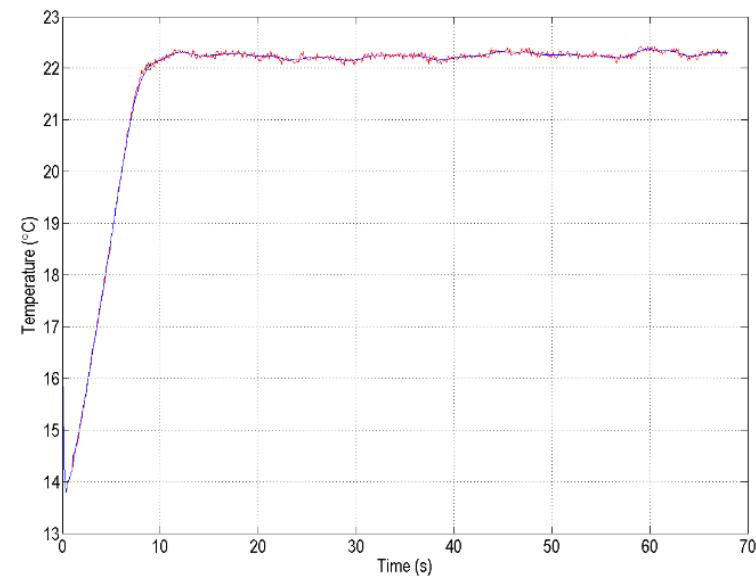

Fig 4. A representative example of transient pixel temperature with (red) and without (blue) filtering.

The criterion used for pixel drying time is seen in Eq. (1). 


$$
t_{d y}=t_{\text {frame }} \text { - wixhen } \frac{\left(T_{\text {frame }}-T_{\text {min }}\right)}{\left(\bar{T}_{\text {plateau }}-T_{\text {min }}\right)} \geq 95 \%
$$

The drying time $t_{d r y}$ was set to the time stamp trame of the first frame fulfilling the criterion. The criterion was set to when the temperature $T_{\text {frame }}$ had increased $95 \%$ of the way from the minimum temperature $T_{\min }$ to the temperature plateau

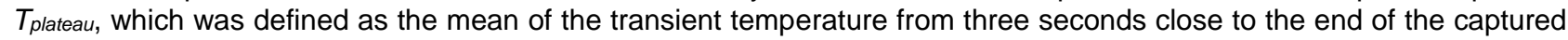
drying sequence. The captured sequence was substantially longer than the expected drying time, ensuring that the three seconds were in the region of level temperature. The $95 \%$ were chosen to further ensure that high frequency noise would not trigger the drying criterion. This resulted in a consistently underestimated drying time, but since only the relative differences in drying time were of interest this did not influence the results.

\subsection{Samples}

The paper samples were made on a Finnish lab sheet former in accordance with an ISO standard (5269-1). Grammages ranged between 15 and $60 \mathrm{~g} / \mathrm{m}^{2}$ with the lower part of the range being relevant for tissue making. The higher grammages were included to enable comparison with standards in the paper making industry, often measured at $\sim 60 \mathrm{~g} / \mathrm{m}^{2}$.

The samples were made from different wood species, all used commercially for tissue making. The pulps used were a mix of birch and aspens for the formation trial, and eucalyptus and a spruce and pine mix respectively for the fibre web-fabric interaction trial. All pulps were bleached and chemically separated, meaning the wood was cooked with chemicals to disintegrate them into separate fibres. For the formation trial, samples with intentionally bad formation were made by prolonging the delay time between mixing the pulps and forming them on the lab sheet former by a factor ten.

\subsection{Fabrics}

In the fibre web-fabric interaction trial the fabrics were either of two commercially used TAD fabrics. One generally used for both towel and toilet paper, with coarser yarns (hereby denoted TAD1), and one used for toilet paper, with thinner yarns (TAD2). The fabrics were fairly similar, why no large differences were expected between them. The hypothesis with the trial was that the areas of the sample in contact with the fabric yarns would be blocked off from air flow and thus dry slower. To explore the extremes of this a model surface was also used, with well-defined holes with 2 mm diameter was used. Here the area between the holes was completely blocked from air flow. In the formation trial the permeable surface was a standard forming wire for general paper making. It has a much finer structure than the TAD fabrics and as such would not influence the local drying times to any great extent.

\section{Results and discussion}

\subsection{Formation}

The local drying time was visualised in contour plots. This basically became a drying time map of the paper sample, where the pixel coordinates represented a specific part of the sample. The colour indicated how long time it took for that specific area to dry. A representative example of a drying time map of a sample with good formation is seen in figure 5 .

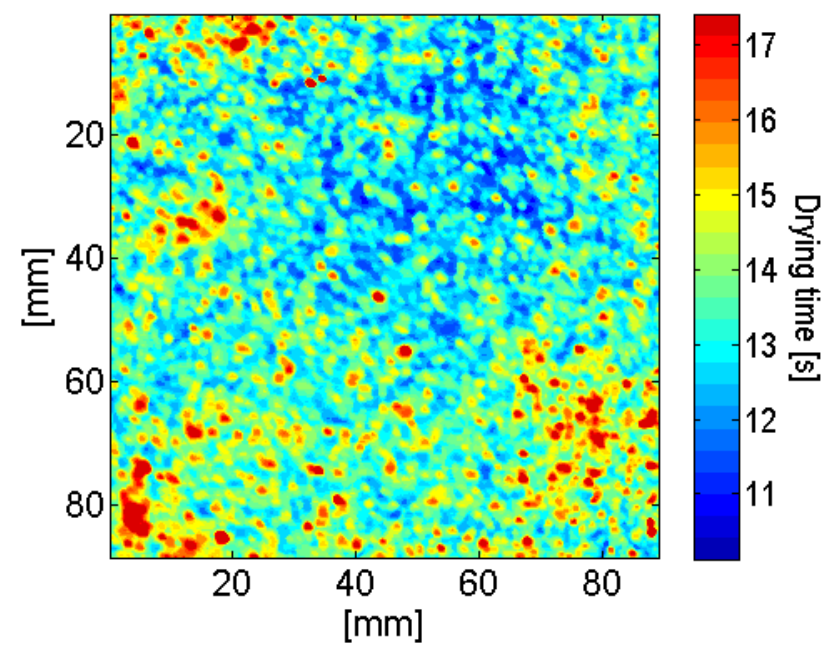

Fig 5. Example of a drying time map of a hardwood sample with $45 \mathrm{~g} / \mathrm{m}^{2} \mathrm{grammage.}$ 
The pattern visible in the figure is a consequence of the non-uniformity of drying. The grainy pattern indicated that the length-scale of the variations were $\sim 2-3 \mathrm{~mm}$. This is slightly larger than the individual fibres and as such likely connected to fibre flocs. The pore size distribution is smaller than the pixel resolution, which somewhat obfuscates the impact of local variations in air flow.

The samples with bad formation behaved differently, which a representative example of can be seen in figure 6 .

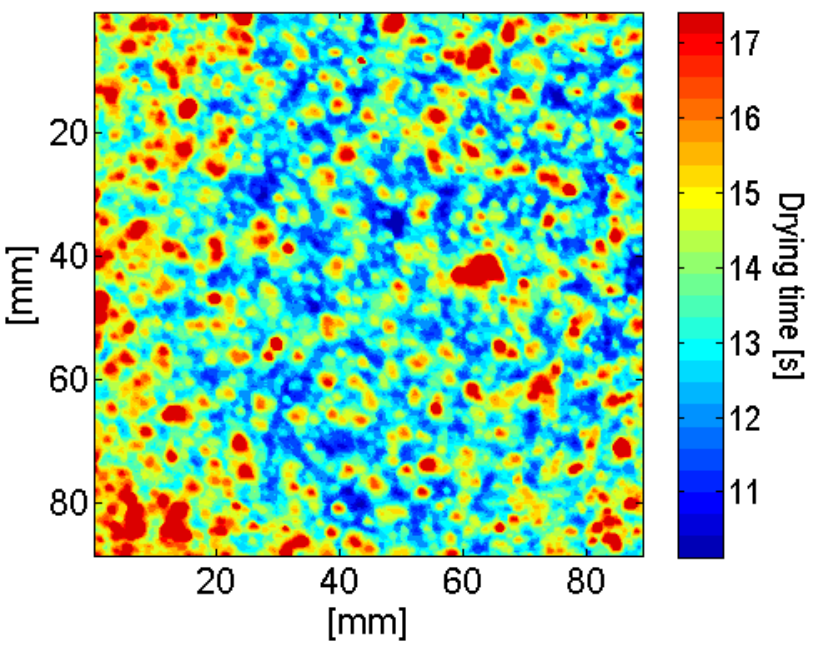

Fig 6. Example of drying time map of a HW sample with $45 \mathrm{~g} / \mathrm{m}^{2}$ grammage and bad formation.

The pattern, while similar, still consistently differed for the samples with bad formation. The length-scale of the grainy pattern was longer, typically ranging from $\sim 5-10 \mathrm{~mm}$. This was attributed to the severe flocculation of the fibres in the samples with bad formation. When the delay time after mixing was increased to such a large extent, the fibres had very long time to collide and get tangled up with other fibres. Interestingly, the change in pattern did not have any significant effect on the total drying time of the sample. Since paper drying must be dimensioned to accommodate the drying of the slowest drying part of the paper, it could be considered a positive result. However, in the literature, the opposite has been found $[11,12,13]$. The differences between this trial and those in the literature was the ingoing dryness. These samples were rather dry, and thus most of the water was located inside the fibre wall. The inter-fibre pores were open already at the beginning of the drying sequence, which presumably limits the impact of non-uniform air flow. The visual differences in the drying time maps were mirrored in the transient coefficient of variation of surface temperature over the entire analysed area. The samples with bad formation had 1.5-2 \% higher coefficient of variation consistently for all grammages.

\subsection{Fibre web-fabric interaction}

When the fibre web-fabric interaction was investigated the model surface was considered first. A representative example of a drying time map of a eucalyptus sample dried on the model surface is seen in figure 7 .

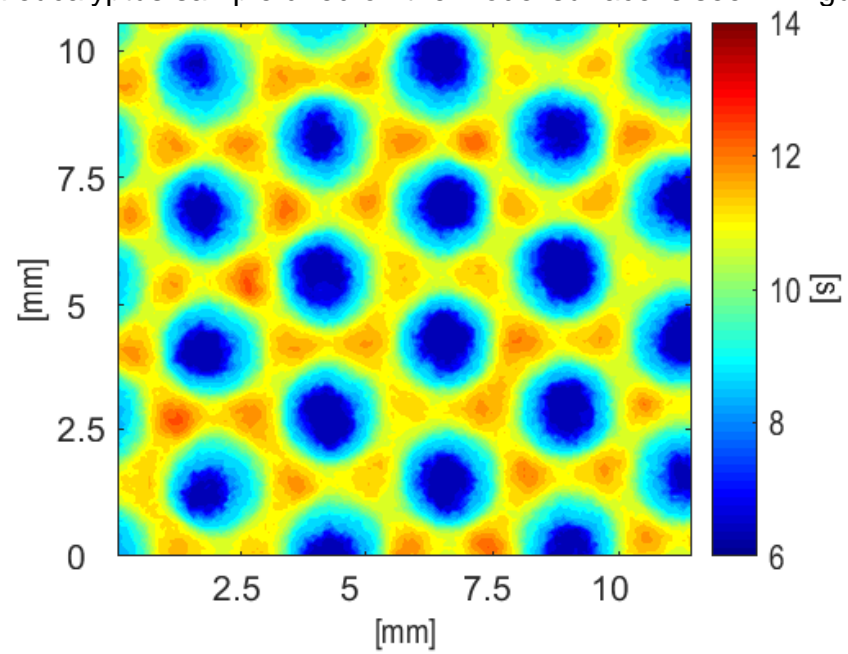

Fig 7. Example of a drying time map of a hardwood sample with $15 \mathrm{~g} / \mathrm{m}^{2}$ grammage on the model surface.

It was immediately evident that the areas blocked from air flow dried out slower than the areas over the holes, but there was also a gradient in drying time from the centre of the holes to the middle of the blocked area in between two holes. 
This gradient indicated the importance of understanding how air enters the sample surface, and that blocking the air flow on the back side might prolong the required time for drying significantly. The drying time difference was up to 4 seconds over distances shorter than the fibre length. This was found for the hardwood samples made from eucalyptus, but the softwood samples made from a spruce and pine mix showed less drying time difference. This implied that the fibre morphology was important for the non-uniformity of drying as it pertains to fibre web-fabric interaction. The softwood fibres are much coarser and longer, which means that there are much fewer fibres per area at the same grammage. The paper structure is thus more open, with less tortuosity of the air flow path.

Having seen the impact of complete blockage with the model surface, the commercially used TAD fabrics were evaluated. In figure 8 a representative example of a drying time map from a hardwood sample dried on TAD1 is seen.

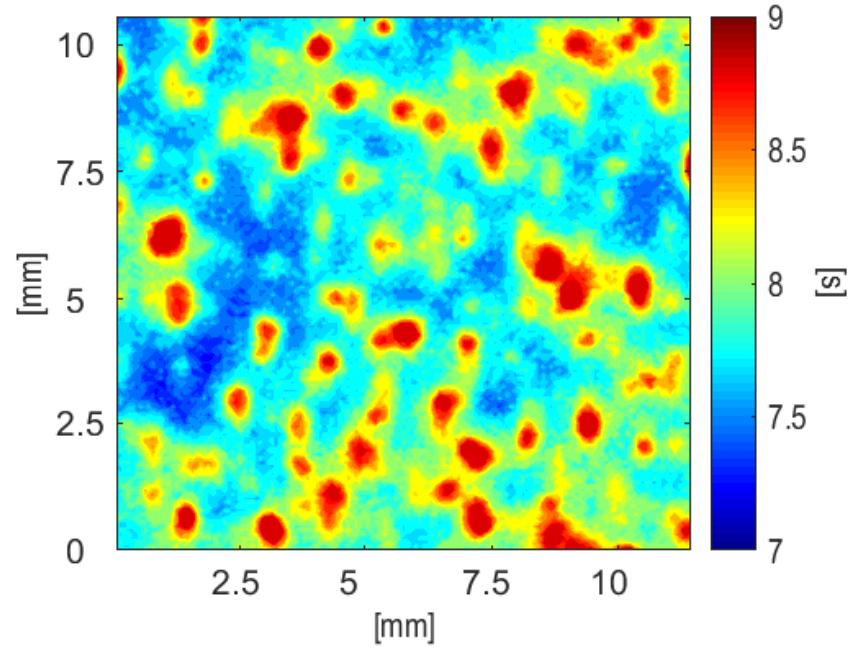

Fig 8. Example of a drying time map of a hardwood sample with $22 \mathrm{~g} / \mathrm{m}^{2}$ grammage on TAD fabric 1.

Compared to the model surface, it was difficult to discern any pattern from the fabric in the drying time map. There were some reoccurring diagonal spots of longer drying times, but it was not clear if this was related to the fabric. Nonuniformities of larger scale than what would be obtained from the fabric showed quite large drying time differences, which obfuscated the small-scale patterns expected from the fabric. To remedy this, a $9 \times 9$ pixels median filter was applied. The size was chosen to represent roughly two times the fabric yarn thickness. The filtered image thus only contained variations larger than the fabric yarn. This image was subtracted from the original drying time map, leaving only the deviation of the small-scale variation. A representative example of a drying time deviation map of a hardwood sample is seen in figure 9.

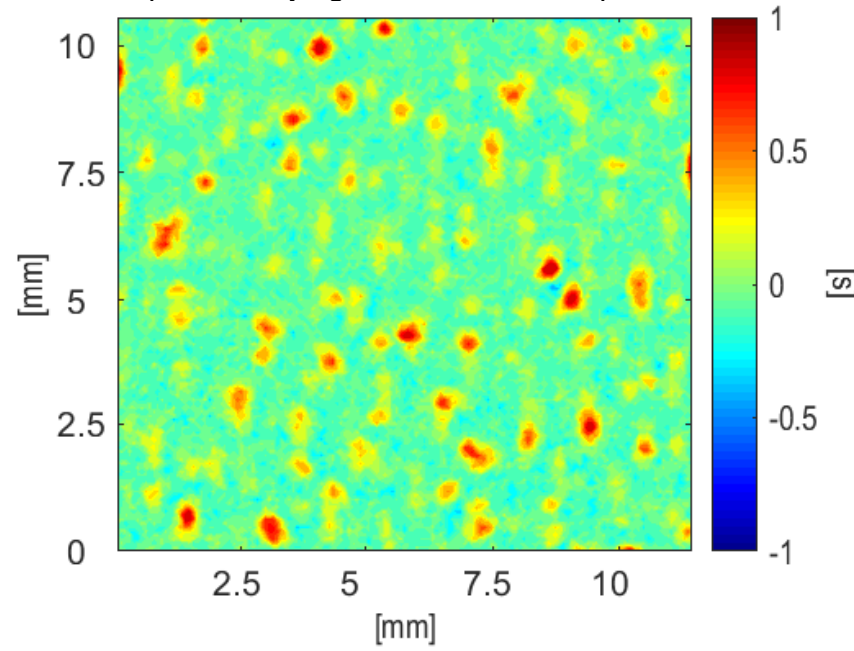

Fig 9. Example of a drying time deviation map of a hardwood sample with $22 \mathrm{~g} / \mathrm{m}^{2}$ grammage on TAD fabric 1.

The diagonal orientation of the spots of longer drying time became more apparent. They correlated well with the highest knuckles of the yarns in the fabric weave. Between the more distinct spots there were also some more oval spots, which correlates well with the second highest knuckles of the fabric, which have a larger area. The increase in drying time at the knuckles was close to $10 \%$ of the total drying time. It was also noted that while spots with longer drying time was obtained, there were not any concentrated areas of shorter drying times, which could have been expected if the air flow would result in the same gradient as seen for the model surface. The air flow thus appeared to be fairly uniform for all areas except the knuckles. It should be noted that other small-scale variations in drying time were not removed by the filter, thus the pattern could not be expected to line up perfectly with the weave pattern of the fabric. 
Just as for the model surface, there were differences between the different pulps used. A representative example of a drying time deviation map of a softwood sample is seen in figure 10.

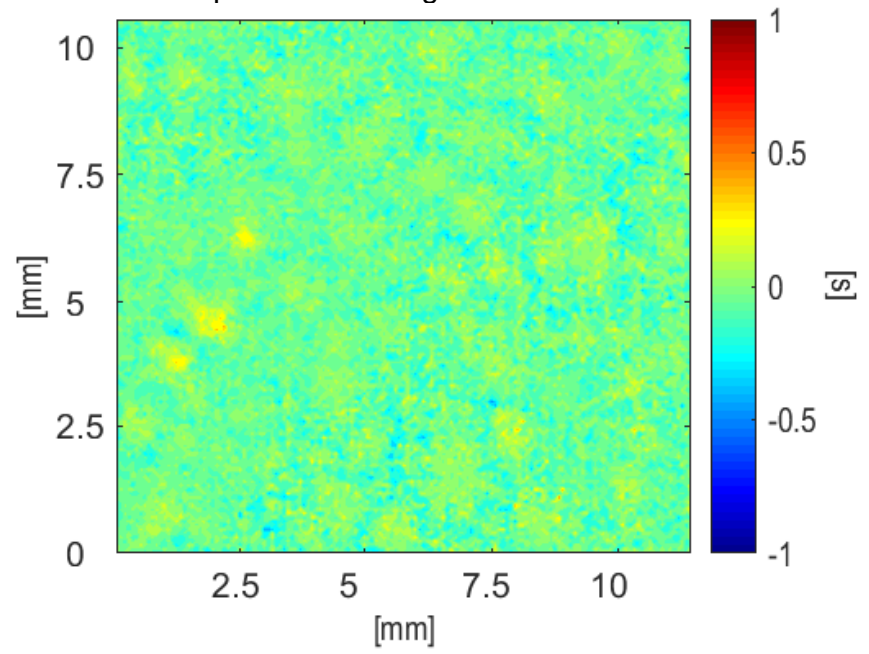

Fig 10. Example of a drying time deviation map of a softwood sample with $22 \mathrm{~g} / \mathrm{m}^{2} \mathrm{grammage}$ on TAD fabric 1.

Here, it was even more difficult to discern any pattern. For the model surface, the drying time differences was only reduced for the softwoods samples, but still present. On the TAD fabrics there were virtually no pattern from the fabric in the softwood samples. It could also be mentioned here, that as expected, there were no observable differences between the TAD fabrics.

\section{Conclusions}

A thermographic method was developed, where the non-uniformity of through air drying in tissue paper making was investigated. The influence of the formation of paper samples and the fibre web-fabric interaction on the non-uniformity was considered. The method allowed for high spatial resolution determination of local drying times of samples on a piece of laboratory drying equipment.

It was found that the formation severely impacted the non-uniformity but that it did not have a detrimental influence on the total drying times of the samples. This surprising finding was believed to be attributed to the high ingoing dryness of samples.

The fibre web-fabric interaction was found to cause prolonged drying times of up to $10 \%$ of the total drying time. On a model surface it was shown that blocking parts of the sample to air flow could create great differences in drying time on length-scales shorter than the fibre length. On commercially used through air drying fabrics the knuckles of the fabric weave were found to be the main cause of prolonged drying times. It was also found that the morphology of the samples was paramount for the severity of the web-fabric interaction. Softwood samples, with coarser fibres showed very little, if any, influence of the interaction. The hardwood samples consistently showed variations in drying time.

\section{REFERENCES}

[1] Norman, B., Pappersteknik, Institutionen för pappersteknik - Kungliga Tekniska Högskolan, 1991.

[2] Gavelin, G., Söder, I. and Jonsson, B., Mjukpapper, Skogsindustrins Utbildning i Markaryd AB, 1999.

[3] Heikkilä, P. and Paltakari, J., Papermaking Part 2, Drying, Paper Engineers' Association/Paperi ja Puu Oy, Helsinki, pp. 39-77, 2010.

[4] Klerelid, I. and Milosavljevic, N., Papermaking Part 2, Drying, Paper Engineers' Association/Paperi ja Puu Oy, Helsinki, pp. 164-209, 2010.

[5] Weineisen, H., Through Drying of Paper - A Literature Review, A40, Svensk Gastekniskt Center, Malmö, Sweden, 2007.

[6] Norman, B. and Wahren, D., A comprehensive method for the description of mass distribution in sheets and flocculation and turbulence in suspensions, Svensk Papperstidning - Vol. 75, no 20, pp. 807-818, 1972.

[7] Janda, B., Sheet Structure Process Effect on Tissue Properties, PaperCon, Nashville, pp. 3231-3253, 2014.

[8] Hyll, K., Image-based quantitative infrared analysis and microparticle characterisation for pulp and paper applications, Ph.D. Thesis, KTH, Stockholm, Sweden, 2016.

[9] Tysén, A., Through air drying - The influence of formation and pulp type on non-uniform drying and air flow, Licentiat of Engineering Thesis, Karlstad University, Universitetstryckeriet, Karlstad, 2014.

[10] Tysén, A. and Vomhoff, H., Method for the quantification of in-plane drying non-uniformity, Nordic Pulp \& Paper Research Journal - Vol. 30, no 2, pp. 286-295, 2015. 
[11] Gomes, V. G., Crotogino, R. H. and Douglas, W. J. M., The Role of Local Nonuniformity in Through Drying of Paper, International Drying Symposium, Montreal, pp. 994-1006, 1992.

[12] Hashemi, S. J. and Douglas, W. J. M., Comparative Techniques For Characterizing Drying Nonuniformity in Drying of Paper, International Drying Symposium, São Paulo, pp. 1303-1310, 2004.

[13] Zuo, H., Modak, A., Ryan, M. and Ramaswamy, S., Experimental analysis of the effect of local non-uniformity on convective heat and mass transfer in porous media, 14th International Drying Symposium, São Paulo, Brazil, pp. 1311-1318, 2004. 